\title{
Measurements of thickness for metallic plates with co-axial holes using a novel analytical method with the modified integration range
}

\author{
Wuliang Yin ${ }^{1}$, Ruocheng Huang ${ }^{2,}$, Mingyang Lu ${ }^{2,}{ }^{\star}$, Zhijie Zhang ${ }^{1}$, Anthony Peyton ${ }^{2}$ \\ ${ }^{1}$ School of Instrument and Electronics, North University of China, Taiyuan, Shanxi, 030051 China \\ ${ }^{2}$ School of Electrical and Electronic Engineering, University of Manchester, Manchester, M13 9PL UK \\ Corresponding author: Mingyang Lu (mingyang.lu@manchester.ac.uk), Ruochen Huang (ruochen.huang@ manchester.ac.uk).
}

\begin{abstract}
The existence of the hole on a plate affects the calculation of eddy current problems. Consequently, the accuracy for the prediction of the material properties decreases if the effect of the hole is not taken into account. In this paper, a novel analytical method based on the modified integration range is proposed which can address the presence of the hole. Due to the presence of the hole, the conventional Dodd-Deeds analytical solution cannot be used to calculate the inductance change. Therefore, a revised upper integration limit is introduced to replace the original limit -- $\infty$ when using the co-axially air-core electromagnetic sensor. With the presence of the hole, the magnitude of the received signal reduces, and the peak frequency feature changes. The analytical method is validated by measured and numerical simulation results. It is found that the upper limit is related to the radius of the open hole. With the new technique, the thickness of sample plates with holes can be estimated based on the peak frequency feature.
\end{abstract}

INDEX TERMS Magnetic induction, eddy current testing, thickness measurement.

\section{INTRODUCTION}

The measurements using the induced eddy current field in the tested material have become a promising technique in the field of non-destructive testing. Eddy current (EC) testing takes advantage of non-contacting to the sample with high accuracy and efficiency. In the industrial area, the significance of EC has been found in many applications, i.e. the thickness measurement [1-3], crack detection [4-5] and non-destructive welding [6-7].

In the field of electromagnetic (EM) sensing, three types of eddy current measurement methods are frequently used for various purposes in industry, that is, pulsed eddy current (PEC) testing, single-frequency eddy current (SEC) testing, and multi-frequency eddy current (MEC) testing. By using PEC technique, the transformer approximation model proposed by $\mathrm{Kral}$, et al. proved its robust to eliminate the effect of lift-off for the thickness measurement [8]. Besides, the lift-off information can be inversed by the relative variation of magnetic flux. Although it is sensitive to the electromagnetic properties of the ferromagnetic plate, it is suitable to evaluate the thermal insulation thicknesses for most cases [9].
Instead of injecting a rectangular stimulus signal to the exciting coil of the sensor probe, multi-frequency eddy current testing technique uses the sinusoidal signals as excitation signals to detect the change of the eddy current under different frequencies. Rich information and better Signal-to-Noise Ratio (SNR) can be received for the use of electromagnetic property prediction and lift-off elimination, etc. The multi-frequency eddy current testing technique to monitor the production of the steel has been explored [10-12]. Various approaches have been proposed to monitor the thickness of tested samples, which mainly involves different sensor designs, measurement techniques and data-processing methods [13-15]. Considering the arrangements of sensor coils, a novel helix triple electromagnetic sensor was designed, and it is found out that thickness estimation can be carried out virtually immune to lift-off variations [16]. For non-magnetic metal plate, the phase signature measured by the electromagnetic sensor was investigated and insensitive to the lift-off. Based on this fact, the thickness can be measured, with the error controlled within 5\% [17]. Besides, Cheng found that the maximum/minimum phase value of the normalized impedance was corresponded to a specific 
thickness. By summarizing and using this relationship as a pre-prepared master curve, the thickness of the tested sample can be obtained without knowing its conductivity [18]. Mao and Lei used the Levenberg-Marquardt algorithm to solve the inversion problem for the pipe and determine the thickness of the metal pipe [15]. Moreover, the compensation algorithm proposed by $\mathrm{Lu}$ et al. can be used to measure the sample thickness but also tackle the effect of the lift-off [19]. However, the presence of the hole on the sample plate can affect the accuracy of the measurements. For this case, it is commonly simulated by FEM. Although analytical methods have been used to evaluate the sample properties due to the defect, it only applies to the infinitely long crack [20].

In this paper, concerning the effect caused by the open hole on the sample plate, we propose a novel analytical method by introducing a revised upper limit constraint to denote the influence of the hole. The inductance change due to the existence of the hole in the sample plate can be obtained via the air-core sensor. The tested sample should be located co-axially to the sensor probe. The mathematical calculation is validated by experiments. Further, thickness measurements have been carried out by utilizing the proposed method.

\section{Sensor setup}

Figure 1 demonstrates the geometry of the air-core sensor. The sensor consists of one exciting coil and one receiving coil. These two coils are co-axially placed above the sample plate with identical coil parameters, i.e. turns, sensor radius, height. The detailed parameters are listed in Table 1. In the simulation setup, the sensor probe with the inner radius of 28 $\mathrm{mm}$ and the outer radius of $28.25 \mathrm{~mm}$ was used to validate the proposed method. Each coil winding has 30 turns. A smaller sized sensor with the turns of 20 was designed to measure the thickness of the sample plate with a hole. The inner and outer radii of the sensor probe are $12.5 \mathrm{~mm}$ and $12.7 \mathrm{~mm}$, respectively.

TABLE I

SENSOR PARAMETERS

Inner radius of the excitation/receiving coil $\left(r_{1}\right)$

Outer radius of the excitation/receiving coil

$\left(r_{2}\right)$

Height of the excitation/receiving coil $\left(h_{e} / h_{p}\right)$

Number of turns $(\mathrm{N})$

\begin{tabular}{c}
$12.5 \mathrm{~mm}$ \\
$28 \mathrm{~mm}$ \\
$12.7 \mathrm{~mm}$ \\
$28.25 \mathrm{~mm}$ \\
$5 \mathrm{~mm}$ \\
$8 \mathrm{~mm}$ \\
20 \\
30 \\
$5 \mathrm{~mm}$ \\
$1 \mathrm{~mm}$ \\
$1 \mathrm{~mm}$ \\
$22 \mu \mathrm{m}-88 \mu \mathrm{m}$ \\
$10 \mathrm{~mm}, 20 \mathrm{~mm}, 25$ \\
$\mathrm{~mm}, 30 \mathrm{~mm}$ \\
\hline
\end{tabular}

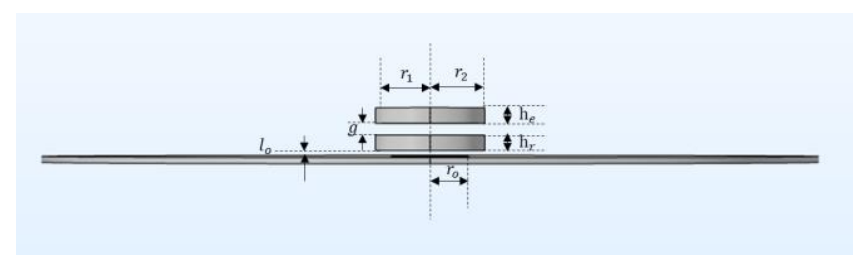

FIGURE 1. Sensor geometry

\section{Analytical calculation of inductance change due to the effect of a co-axial hole}

As a crucial indicator, the inductance change between the excitation coil and receiving coil has been widely used in various applications in the field of non-destructive testing. From our previous researches, the peak frequency feature of the imaginary part of the inductance change varies under different sample conductivities and thicknesses. As a theoretical basis, the analytical solution proposed by Dodd and Deeds proved its efficiency and accuracy in obtaining the inductance change due the presence of the sample plate. However, by substituting the boundary conditions of the magnetic field for each region, it is also capable to calculate the impedance change considering the effect of the hole but is troublesome. Thus, a novel analytical method by introducing an upper constraint is proposed in this paper.

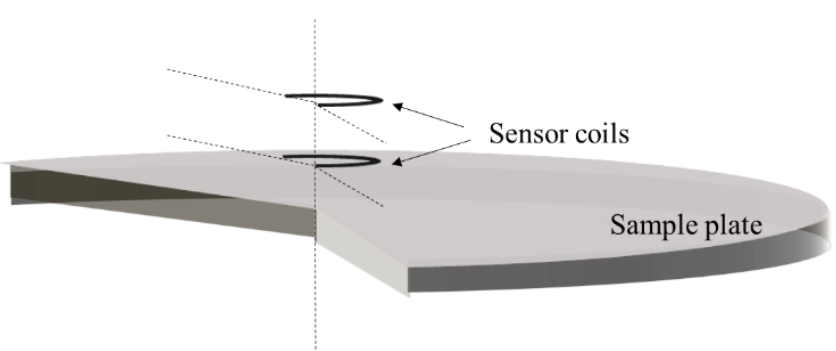

FIGURE 2. Dodd-Deeds model for the coil coaxially above the infinite sample plate

Figure 2 demonstrates the Dodd-Deeds model for calculating the sensor coils above the infinite large sample plate. In general, the sample satisfying the condition that the radius (or size) is over 3-5 times the sensor coil radius can be regarded as an infinite sample plate. Consequently, the inductance change due to the existence of the sample plate can be obtained by using equations (1)-(6). It can be noticed that the integration domain for an infinite plate is from 0 to infinity as shown in equation (1).

$$
\begin{aligned}
& \Delta \mathrm{L}(\omega)=\frac{\left.N \mathrm{j} \omega \int_{\mathrm{S}} \mathrm{Ads}\right|_{\sigma, \mu_{1}}-\left.\mathrm{Nj} \omega \int_{\mathrm{S}} \mathrm{Ads}\right|_{\sigma=0, \mu_{0}}}{\mathrm{j} \omega \mathrm{I}}= \\
& \frac{\pi \mathrm{r}_{0} \mathrm{~N}^{2} \mu_{0}}{\left(\mathrm{l}_{\mathrm{s} 1}-\mathrm{l}_{\mathrm{s} 2}\right)^{2}\left(\mathrm{r}_{s 2}-\mathrm{r}_{s 1}\right)^{2}} \int_{0}^{\infty} \frac{\mathrm{P}^{2}(\alpha)}{\alpha^{6}} \mathrm{e}^{-\alpha\left(2 l+\mathrm{l}_{\mathrm{e} 2}-\mathrm{l}_{\mathrm{e} 1}+\mathrm{g}\right)}\left(1-\mathrm{e}^{-2 \alpha\left(\mathrm{l}_{\mathrm{e} 2}-\mathrm{l}_{\mathrm{e} 1}\right)}\right) \phi(\alpha) \mathrm{d} \alpha \\
& (1) \\
& \mathrm{A}(\mathrm{r}, \mathrm{z})=\frac{\mathrm{I} \mu_{0} N}{2} \int_{\alpha_{r_{s}}}^{\infty} \frac{\mathrm{J}_{1}(\alpha \mathrm{r}) \mathrm{P}(\alpha)}{\alpha^{3}} \mathrm{~K}(\mathrm{r}, \mathrm{z}, \alpha) \phi(\alpha) \mathrm{d} \alpha \\
& \phi(\alpha)=\frac{\left(\alpha_{1}+\mu_{1} \alpha\right)\left(\alpha_{1}-\mu_{1} \alpha\right)-\left(\alpha_{1}+\mu_{1} \alpha\right)\left(\alpha_{1}-\mu_{1} \alpha\right) \mathrm{e}^{2 \alpha_{1} \mathrm{D}_{0}}}{-\left(\alpha_{1}-\mu_{1} \alpha\right)\left(\alpha_{1}-\mu_{1} \alpha\right)+\left(\alpha_{1}+\mu_{1} \alpha\right)\left(\alpha_{1}+\mu_{1} \alpha\right) \mathrm{e}^{2 \alpha_{1} \mathrm{D}_{0}}} \\
& \alpha_{1}=\sqrt{\alpha^{2}+\mathrm{j} \omega \sigma \mu_{1} \mu_{0}}
\end{aligned}
$$


$\mathrm{K}(\mathrm{r}, \mathrm{z}, \alpha)=2-\mathrm{e}^{-\alpha\left(\mathrm{z}-\mathrm{l}_{\mathrm{e} 2}\right)}-\mathrm{e}^{-\alpha\left(\mathrm{z}-\mathrm{l}_{\mathrm{e} 1}\right)}+\mathrm{e}^{-\alpha \mathrm{z}}\left(\mathrm{e}^{-\alpha \mathrm{l}_{\mathrm{e} 1}}-\mathrm{e}^{-\alpha \mathrm{l}_{\mathrm{e} 2}}\right)$

$P(\alpha)=\int_{\alpha \mathrm{r}_{\mathrm{e} 1}}^{\alpha \mathrm{r}_{2}} \tau \mathrm{J}_{1}(\tau) \mathrm{d} \tau$

Where: $\mathrm{N}$ denotes the number of turns of the excitation/receiving coil, $\omega$ denotes the exciting frequency, $\sigma$ denotes the electrical conductivity of the sample plate, $\mu_{0}$ denotes the magnetic permeability in vacuum, $\mu_{1}$ denotes the relative permeability of the sample plate, I denotes the current injected into the excitation coil, $r_{\mathrm{s} 1}$ and $\mathrm{r}_{\mathrm{s} 2}$ denote the inner and outer radii of the receiving coil, $\mathrm{l}_{\mathrm{e} 1}$ and $\mathrm{l}_{\mathrm{e} 2}$ denote the lower and upper height of the excitation coil, $\mathrm{l}_{\mathrm{s} 1}$ and $\mathrm{l}_{\mathrm{s} 2}$ denote the lower and upper height of the receiving coil, $\mathrm{l}_{\mathrm{s} 1}$ and $l_{\mathrm{s} 2}$ denote the lower and upper height of the receiving coil, $\mathrm{D}_{0}$ denotes the thickness of the sample plate, $\mathrm{J}_{1}$ denotes the first-order Bessel function of the first kind.

Paper [21] exhibits the deviations of the Dodd - Deeds model. According to [21], the magnetic field vector potential A produced by the excitation coil is met with the differential equation, which gives

$\frac{\partial^{2} \mathrm{~A}}{\partial \mathrm{r}^{2}}+\frac{1}{\mathrm{r}} \frac{\partial \mathrm{A}}{\partial \mathrm{r}}+\frac{\partial^{2} \mathrm{~A}}{\partial \mathrm{z}^{2}}-\frac{\mathrm{A}}{\mathrm{r}^{2}}+\omega^{2} \mu_{\mathrm{i}} \sigma_{\mathrm{i}} \mathrm{A}-\mathrm{j} \omega \mu_{\mathrm{i}} \sigma_{\mathrm{i}} \mathrm{A}=0$

Assume that the magnetic field contains the $\mathrm{R}$ component and the $\mathrm{Z}$ component, which expresses

$\mathrm{A}=\mathrm{R}(\mathrm{r}) \times \mathrm{Z}(\mathrm{z})$

Combine equation (7) and equation (8), which equals

$\frac{1}{\mathrm{R}(\mathrm{r})} \frac{\partial^{2} \mathrm{R}(\mathrm{r})}{\partial \mathrm{r}^{2}}+\frac{1}{\mathrm{rR}(\mathrm{r})} \frac{\partial \mathrm{R}(\mathrm{r})}{\partial \mathrm{r}}+\frac{1}{\mathrm{Z}(\mathrm{z})} \frac{\partial^{2} \mathrm{Z}(\mathrm{z})}{\partial \mathrm{z}^{2}}-\frac{1}{\mathrm{r}^{2}}+\omega^{2} \mu_{\mathrm{i}} \sigma_{\mathrm{i}}-\mathrm{j} \omega \mu_{\mathrm{i}} \sigma_{\mathrm{i}}=0$

Then, assume that $\alpha^{2}$ satisfies

$\frac{1}{\mathrm{Z}(\mathrm{z})} \frac{\partial^{2} \mathrm{Z}(\mathrm{z})}{\partial \mathrm{z}^{2}}-\alpha^{2}+\omega^{2} \mu_{\mathrm{i}} \sigma_{\mathrm{i}}-\mathrm{j} \omega \mu_{\mathrm{i}} \sigma_{\mathrm{i}}=0$

(10)

Equation (9) turns into

$\frac{1}{\mathrm{R}(\mathrm{r})} \frac{\partial^{2} \mathrm{R}(\mathrm{r})}{\partial \mathrm{r}^{2}}+\frac{1}{\mathrm{rR}(\mathrm{r})} \frac{\partial \mathrm{R}(\mathrm{r})}{\partial \mathrm{r}}+\alpha^{2}-\frac{1}{\mathrm{r}^{2}}=0$

(11)

According to [21], the $\mathrm{R}$ component can be presented by the first-order Bessel function of the first kind, $\mathrm{CJ}_{1}(\alpha r)$. Consequently, equation (11) can be expressed as

$(\alpha \mathrm{r})^{2}-1+\frac{\alpha \mathrm{r}}{4 \mathrm{~J}_{1}(\alpha \mathrm{r})}\left(2\left(\mathrm{~J}_{0}(\alpha \mathrm{r})-\mathrm{J}_{2}(\alpha \mathrm{r})\right)-\alpha \mathrm{r}\left(-3 \mathrm{~J}_{1}(\alpha \mathrm{r})+\mathrm{J}_{3}(\alpha \mathrm{r})\right)\right)=0$

Where: $\mathrm{J}_{0}, \mathrm{~J}_{2}$ and $\mathrm{J}_{3}$ are the zero, second and third order Bessel function of the first kind.

As can be seen from equation (12), the value of $\alpha r$ is the solution of equation. Besides, there is an inverse relationship between $\alpha$ and $r$. Therefore, the integration domain is from 0 to $\infty$ as the radius ranging from $\infty$ to 0 for an infinite sample plate, consequently, the integration domain is from 0 to $\alpha_{r_{o}}$ for the radius ranging from $\infty$ to $r_{o}$ for the infinite sample plate with a hole radius of $r_{o}$, as shown in fig. 3 .

Therefore, the inductance change due to the existence of the sample plate with the hole in the center can be calculated as

$$
\Delta \mathrm{L}(\omega)=\frac{\pi \mathrm{r}_{0} \mathrm{~N}^{2} \mu_{0}}{\left(\mathrm{l}_{\mathrm{s} 1}-\mathrm{l}_{\mathrm{s} 2}\right)^{2}\left(\mathrm{r}_{s 2}-\mathrm{r}_{s 1}\right)^{2}} \int_{0}^{\alpha_{r_{o}}} \frac{\mathrm{P}^{2}(\alpha)}{\alpha^{6}} \mathrm{e}^{-\alpha\left(2 l+\mathrm{l}_{\mathrm{e} 2}-\mathrm{l}_{\mathrm{e} 1}+\mathrm{g}\right)}(1
$$

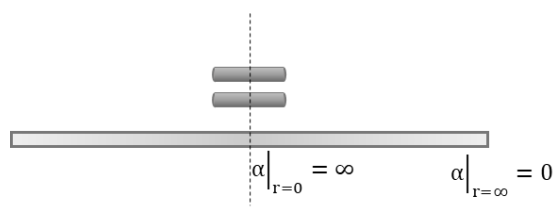

(a)

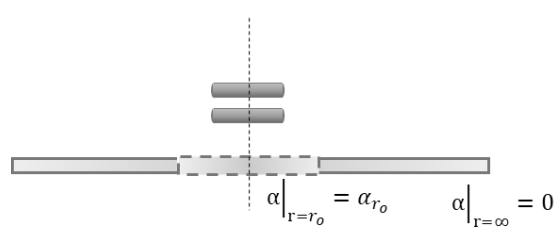

(b)

FIGURE 3. The integration domain of (a) infinite sample plate (b) infinite sample plate with a hole

\section{Simulations and thickness measurement}

\section{A. Simulations}

In the simulation, the sensor with the inner and outer radii of $28 \mathrm{~mm}$ and $28.25 \mathrm{~mm}$ was used to measure the change of the inductance due to the sample plate with a hole. The conductivity and the thickness of the sample plate was set to $32 \mathrm{MS} / \mathrm{m}$ and $1 \mathrm{~mm}$ respectively. Since the sample radius is approx. 6 times larger than the sensor radius (normally approx. 3-5 times large), the sample plate can be regarded as an infinite sample plate and can be applied with the analytical solution.

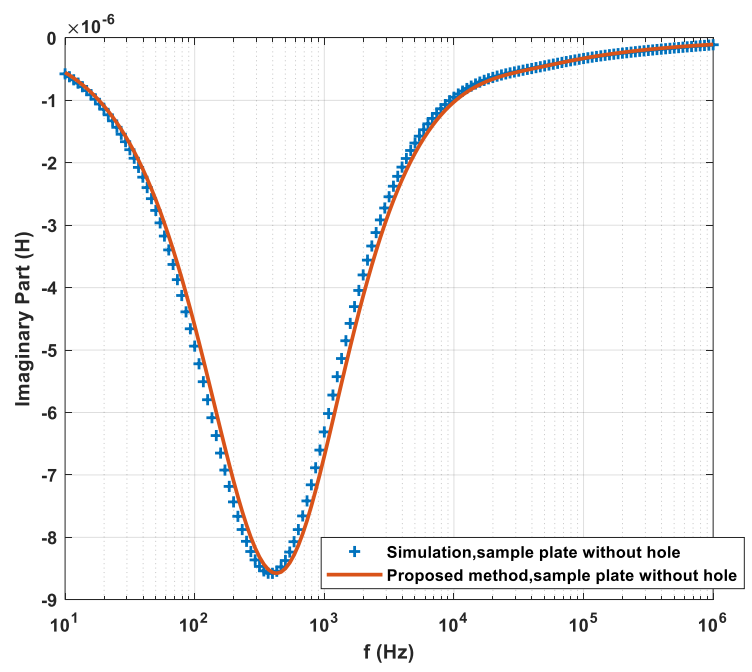

(a) 


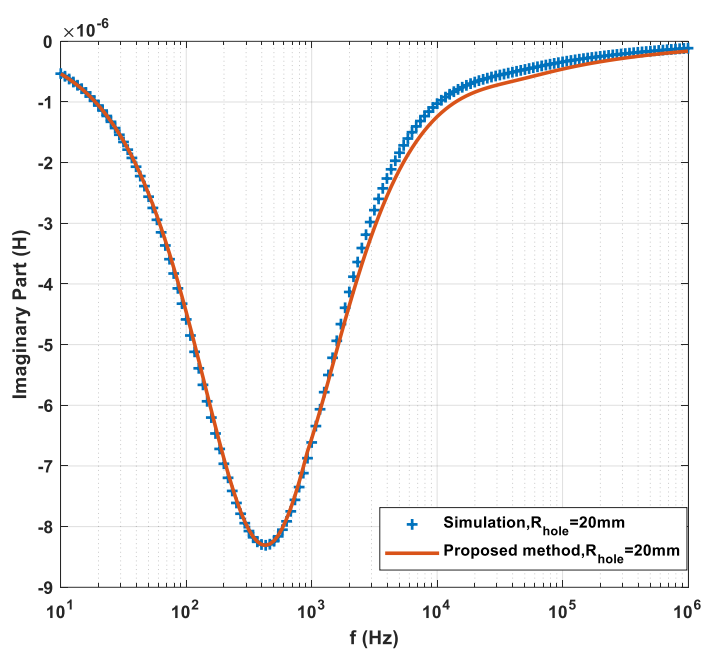

(b)

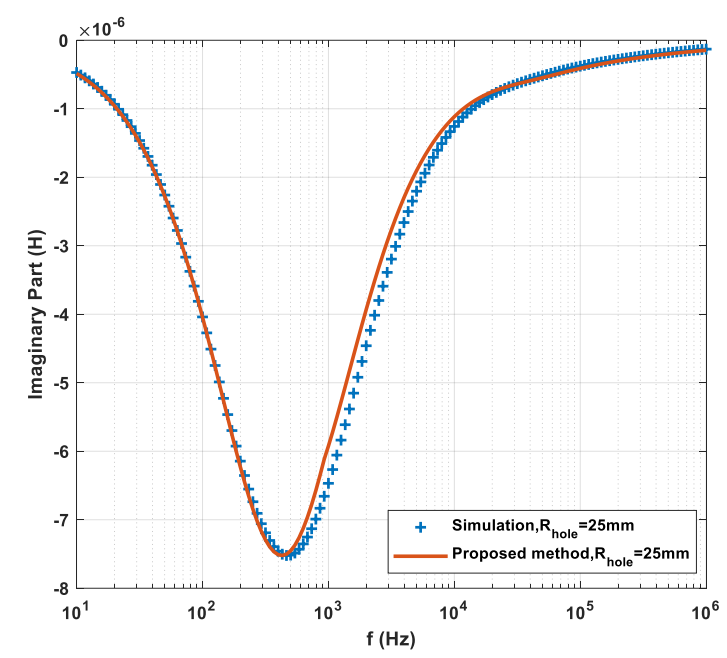

(c)

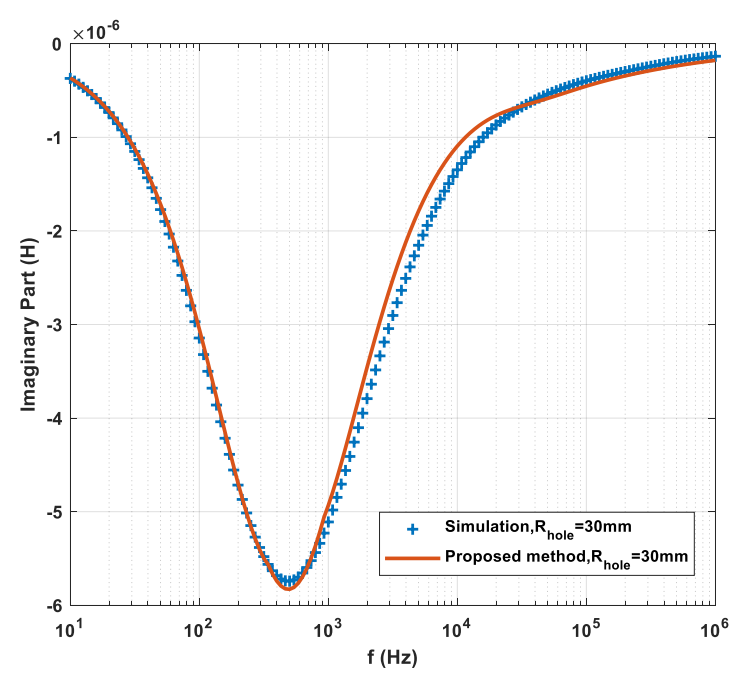

(d)

FIGURE 4. The results of the imaginary part of inductance change (a) sample plate without hole (b) sample plate with the hole radius of 20 $\mathrm{mm}$ (c) sample plate with the hole radius of $25 \mathrm{~mm}$ (d) sample plate with the hole radius of $30 \mathrm{~mm}$
Figure 4 shows the results of the imaginary part of the inductance change under the sample plate with different radii of holes. It can be seen that there is a decreasing trend of the imaginary part of the inductance change under it reaches its minimum where the corresponding frequency is termed as peak frequency, then it increases as the frequency continue to increase. Compared with the simulation results, the results from the proposed method is well agreed with the simulation results. As the hole radius increases, the magnitude of the inductance change reduces with a larger peak frequency. The error under higher frequency may be due to the skin depth effect and the entire mesh domain of the simulation.

\section{B. Thickness measurements}

\section{1) Experimental setup}

The measurements have been carried out to measure the thickness of the sample plate with a hole. Figure 5 exhibits the experimental setup of thickness measurement. The Zurich impedance analyzer was used to test the mutual impedance between the excitation coil and the receiving coil with and without the test piece. The host PC is connected to the impedance analyzer to set parameter and receive the signal data. The current with the amplitude of $0.3 \mathrm{~A}$ was injected into the excitation coil and the sweeping frequency mode was chosen for the tests. The range of the sweeping frequency is from $1 \mathrm{kHz}$ to $510 \mathrm{kHz}$. The inductance change $(\Delta L)$ can be obtained by using the following equations,

$$
\begin{aligned}
& \operatorname{Re}(\Delta L)=\operatorname{Re}\left(\frac{Z_{s}-Z_{a}}{j \omega}\right) \\
& \operatorname{Im}(\Delta L)=\operatorname{Im}\left(\frac{Z_{s}-Z_{a}}{j \omega}\right)
\end{aligned}
$$

Where: $Z_{s}$ denotes the impedance under the presence of the sample plate, $Z_{a}$ denotes the impedance in the air.

In the measurements, the sensor parameters are listed in Table I, the sensor with the radius of $12.5 \mathrm{~mm}$ was used to measure the thickness of aluminium plate with the hole radius of $10 \mathrm{~mm}$. The thickness of the aluminium plate ranges from $22 \mu \mathrm{m}$ to $88 \mu \mathrm{m}$ in the step of $22 \mu \mathrm{m}$; and the lift-off of the sensor is kept to $1 \mathrm{~mm}$.

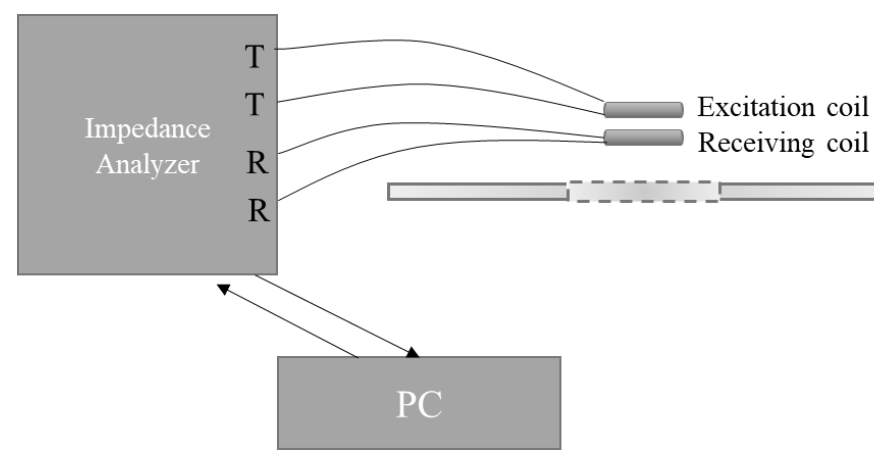

(a) 


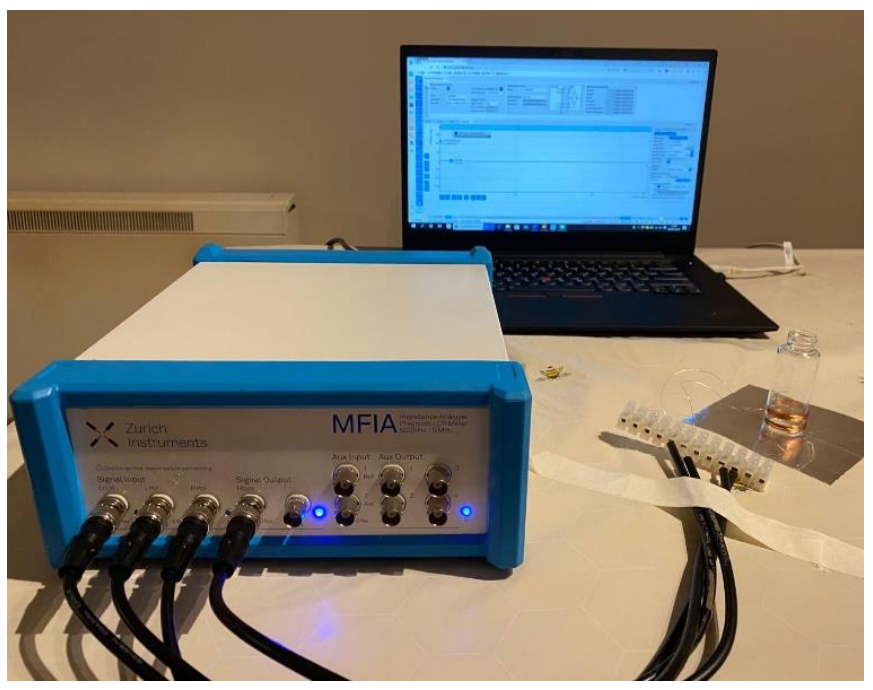

(b)

FIGURE 5. Experimental setup (a) schematic diagram (b) actual setup

2) Results

Figure 6 shows the measurement and analytical results under different thicknesses of the sample plates. There is a decreasing trend for the peak frequency with the increasing thickness of the sample plate. By utilizing the value of the peak frequency, the thickness of the plate can be predicted. From the measurements, the peak frequency from the data stream can be tested by using the curve fitting. Once the peak frequency is obtained, the thickness of the plate can be predicted to find the optimal solution between the proposed method and the measurements.

It can be seen from Fig. 7 that due to the existing hole in the sample plate, the peak frequency increases compared with the sample plate without any cracks. Besides, as shown in Fig. 6, both the measurements and the proposed method fit well with each other. From Table II, the thickness prediction is within a reasonable error (approx. 1\%).

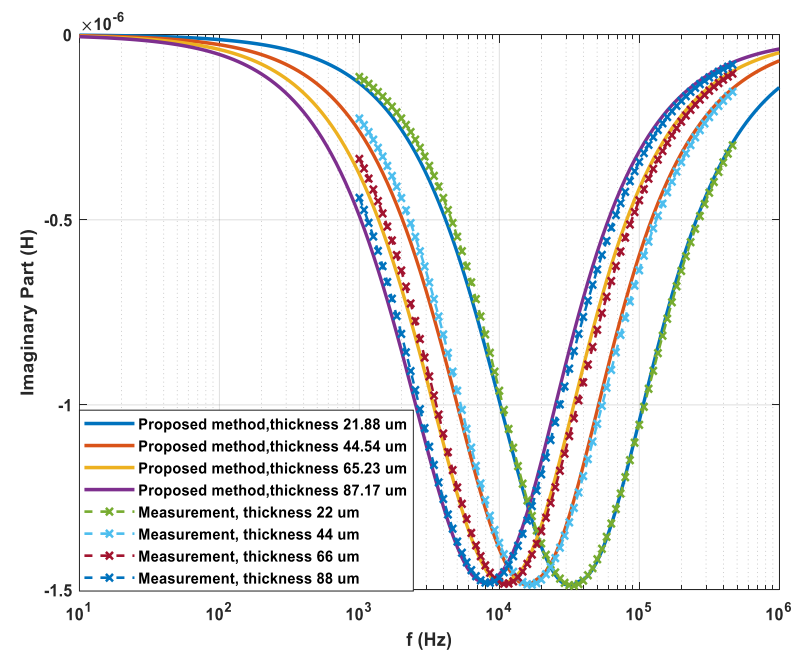

FIGURE 6. Thickness measurement results

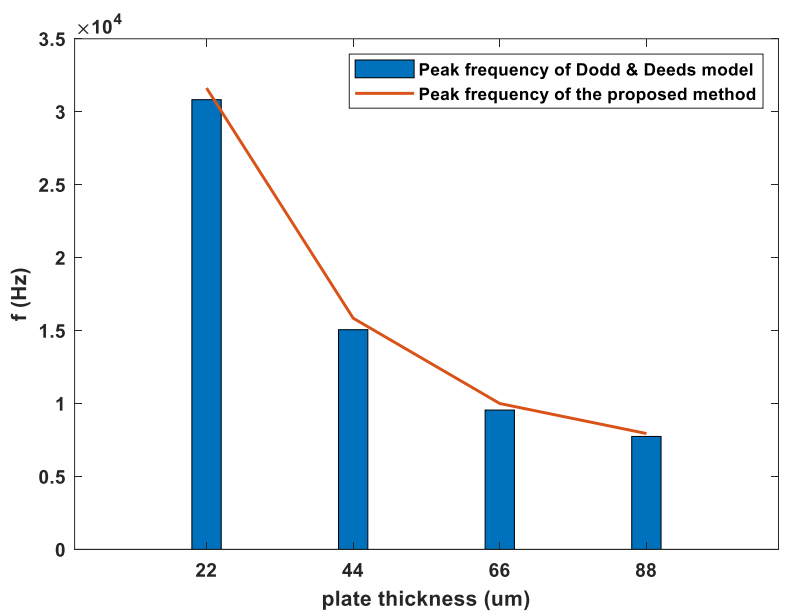

FIGURE 7. Peak frequency under different methods

TABLE II

ACTUAL AND ESTIMATED THICKNESS FOR THE ALUMINIUM PLATES

\begin{tabular}{ccc}
\hline \hline $\begin{array}{c}\text { Actual } \\
\text { thickness } \\
(\mu \mathrm{m})\end{array}$ & $\begin{array}{c}\text { Estimated } \\
\text { thickness } \\
(\mu \mathrm{m})\end{array}$ & Error $(\%)$ \\
\hline 22 & 22.88 & 0.55 \\
44 & 44.54 & 1.23 \\
66 & 65.23 & 1.17 \\
88 & 87.17 & 0.94 \\
\hline
\end{tabular}

\section{CONCLUSION}

In this paper, an analytical method to calculate the inductance change due to the presence of the sample plate with a hole is proposed. By introducing an upper limit, the integration domain is bounded due to the presence of the hole. With this method, the tested sample should be located co-axially to the air-cored co-axially sensor probe. Besides, there is an inversed relation between the upper limit and the hole radius. Moreover, from both simulations and measurements, the peak frequency increases with the hole radius. Further, with the fixed limit, the thickness measurement can be obtained with the error of around $1 \%$.

\section{Acknowledgement}

This work was supported by [UK Engineering and Physical Sciences Research Council (EPSRC)] [grant number: EP/P027237/1] [title: Real-time In-line Microstructural Engineering (RIME)].

\section{REFERENCES}

[1]. Z. Qu, Q. Zhao, Y. Meng, "Improvement of sensitivity of eddy current sensors for nano-scale thickness measurement of $\mathrm{Cu}$ films," NDT \& E International, vol. 61, pp. 53-57, 2014.

[2]. M. Lu, X. Meng, W. Yin, Z. Qu, F.Wu, J. Tang, et al. "Thickness measurement of non-magnetic steel plates using a novel planar triple-coil sensor," NDT \& E International, vol. 107, pp. 102148, 2019.

[3]. M. Lu, L. Chen, X. Meng, R. Huang, A. Peyton and W. Yin, "Thickness measurement of metallic film based on a high-frequency feature of triple-coil electromagnetic eddy current sensor," IEEE Transactions on Instrumentation and Measurement, early access. 
[4]. A. Bernieri, G. Betta, L. Ferrigno, M. Laracca and S. Mastrostefano, "Multifrequency Excitation and Support Vector Machine Regressor for ECT Defect Characterization," IEEE Transactions on Instrumentation and Measurement, vol. 63, no. 5, pp. 1272-1280, May 2014.

[5]. J. Wu, et al., "Surface crack detection for carbon fiber reinforced plastic (CFRP) materials using pulsed eddy current testing," 2014 IEEE Far East Forum on Nondestructive Evaluation/Testing, Chengdu, pp. 181-185, 2014.

[6]. H. Xu, J. R. S. Avila, F. Wu, et al. "Imaging x70 weld cross-section using electromagnetic testing," NDT \& E International, vol. 98, pp. 155-160, 2018.

[7]. B.P.C Rao, B. Raj, T. Jayakumar, and P. Kalyanasundaram, “An artificial neural network for eddy current testing of austenitic stainless steel welds," NDT \& E International, vol. 35, no. 6, pp. 393-398, 2002.

[8]. J. Kral, R. Smid, H. M. G. Ramos and A. L. Ribeiro, "The Lift-Off Effect in Eddy Currents on Thickness Modeling and Measurement," IEEE Transactions on Instrumentation and Measurement, vol. 62, no. 7, pp. 2043-2049, July 2013

[9]. J. Li, X. Wu, Q. Zhang, and P. Sun, "Measurement of lift-off using the relative variation of magnetic flux in pulsed eddy current testing," NDT\&E Int., vol. 75, pp. 57-64, Oct. 2015.

[10]. S. Johnstone, R. Binns, A. J. Peyton, et al. "Using electromagnetic methods to monitor the transformation of steel samples," Transactions of the Institute of Measurement and Control, vol. 23, no. 1, pp. 21-29, 2001.

[11]. S. J. Dickinson, R. Binns, W. Yin, C. Davis, A. J. Peyton, "The development of a multi-frequency electromagnetic instrument for monitoring the phase transformation of hot strip steel," IEEE IMTC, Ottawa, Canada, pp. 1091-1096, 2005.

[12]. W. Yin, S.J. Dickinson, A. J. Peyton, "A multi-frequency impedance analysing instrument for eddy current testing," Measurement Science and Technology, vol. 17, no. 2, pp. 393, 2006.

[13]. F. F. Ren, Y. Lei, "Thickness and Conductivity Measurement of Three-Layered Plane Conductors Based on Harmonic Eddy Current Testing," Nondestructive Testing, vol. 35, no. 8, pp. 50-53, 2013.

[14]. S. J. Norton, J.R. Bowler, "Theory of eddy current inversion," Journal of applied physics, vol. 73, no. 2, pp. 501-512, 1993.

[15]. X. Mao, Y. Lei, "Thickness measurement of metal pipe using swept-frequency eddy current testing," NDT \& E International, vol. 78, pp. 10-19, 2016.

[16]. W. Yin and K. Xu, "A Novel Triple-Coil Electromagnetic Sensor for Thickness Measurement Immune to Lift-Off Variations," IEEE Transactions on Instrumentation and Measurement, vol. 65, no. 1, pp. 164-169, Jan. 2016.

[17]. W. Yin and A. J. Peyton, "Thickness Measurement of Metallic Plates With an Electromagnetic Sensor Using Phase Signature Analysis," IEEE Transactions on Instrumentation and Measurement, vol. 57, no. 8, pp. 1803-1807, Aug. 2008.

[18]. W. Cheng, "Thickness Measurement of Metal Plates Using SweptFrequency Eddy Current Testing and Impedance Normalization," in IEEE Sensors Journal, vol. 17, no. 14, pp. 4558-4569, 15 July15, 2017.

[19]. M. Lu, L. Yin, A. J. Peyton and W. Yin, "A Novel Compensation Algorithm for Thickness Measurement Immune to Lift-Off Variations Using Eddy Current Method," in IEEE Transactions on Instrumentation and Measurement, vol. 65, no. 12, pp. 2773-2779, Dec. 2016.

[20]. T. Theodoulidis, "Analytical model for tilted coils in eddy-current nondestructive inspection," in IEEE Transactions on Magnetics, vol. 41, no. 9, pp. 2447-2454, Sept. 2005.

[21]. C. V. Dodd, and W. E. Deeds. "Analytical solutions to eddy-current probe-coil problems." Journal of applied physics, vol. 39, no. 6, pp. 2829-2838, 1968.
[22]. M. Lu, et al., "Acceleration of frequency sweeping in eddy-current computation," IEEE Transactions on Magnetics, vol. 53, pp. 1-8, 2017.

[23]. M. Lu, R. Huang, W. Yin, Q. Zhao and A. Peyton, "Measurement of permeability for ferrous metallic plates using a novel lift-off compensation technique on phase signature," IEEE Sensors Journal, vol. 19, no. 17, pp. 7440-7446, 1 Sept.1, 2019.

[24]. M. Lu, H. Xu, W. Zhu, L. Yin, et al., "Conductivity Lift-off Invariance and measurement of permeability for ferrite metallic plates," NDT \& E International, vol. 95, pp. 36-44, Apr. 2018.

[25]. M. Lu, et al., "Determination of the magnetic permeability, electrical conductivity, and thickness of ferrite metallic plates using a multi-frequency electromagnetic sensing system," IEEE Transactions on Industrial Informatics, vol. 15, pp. 4111-4119, 2019.

[26]. J. R. S. Avila, M. Lu, et al., "Accurate measurements of plate thickness with variable lift-off using a combined inductive and capacitive sensor," NDT \& E International, vol. 110, pp. 102202, 2020.

[27]. R. Huang, M. Lu, A. Peyton and W. Yin, "Thickness measurement of metallic plates with finite planar dimension using eddy current method," IEEE Transactions on Instrumentation and Measurement, vol. 69, no. 10, pp. 8424-8431, 2020.

[28]. M. Lu, et al., "Measurement of ferromagnetic slabs permeability based on a novel planar triple-coil sensor," IEEE Sensors Journal, vol. 20, no. 6, pp. 2904-2910, 2020.

[29]. M. Lu, W. Zhu, L. Yin, A. J. Peyton, W. Yin and Z. Qu, "Reducing the lift-off effect on permeability measurement for magnetic plates from multifrequency induction data," IEEE Transactions on Instrumentation and Measurement, vol. 67, no. 1, pp. 167-174, Jan. 2018.

[30]. M. Lu, et al., "Measuring lift-off distance and electromagnetic property of metal using dual-frequency linearity feature," IEEE Transactions on Instrumentation and Measurement, early access, 2020. Doi: 10.1109/TIM.2020.3029348.

[31]. M. Lu, et al., "Lift-off tolerant pancake eddy-current sensor for the thickness and spacing measurement of nonmagnetic plates," IEEE Transactions on Instrumentation and Measurement, early access, 2020. DOI: $10.1109 /$ TIM.2020.3033377

[32]. W. Yin, J. Tang, M. Lu, et al., "An equivalent-effect phenomenon in eddy current non-destructive testing of thin structures," IEEE Access, vol. 7, pp. 70296-70307, 2019.

[33]. R. Huang et al., "Measuring Co-Axial Hole Size of Finite-Size Metallic Disk Based on a Dual-Constraint Integration Feature Using Multi-Frequency Eddy Current Testing," IEEE Transactions on Instrumentation and Measurement, early access, 2020. DOI: 10.1109/TIM.2020.3026762

[34]. R. Huang et al., "Measurement of the radius of metallic plates based on a novel finite region eigenfunction expansion (FREE) method," IEEE Sensors Journal, early access, 2020. DOI: 10.1109/JSEN.2020.3009443

[35]. J. Tang et al., "A Novel Efficient FEM Thin Shell Model for BioImpedance Analysis," Biosensors, vol. 10, no. 6, pp. 69, 2020.

[36]. L. Chen, et al., "Textile Based Capacitive Sensor for Physical Rehabilitation via Surface Topological Modification," ACS Nano, vol. 14, no. 7, pp. 8191-8201, 2020. DOI: 10.1021/acsnano.0c01643

[37]. Z. Jin, et al., "Methods of Controlling Lift-off in Conductivity Invariance Phenomenon for Eddy Current Testing," IEEE ACCESS, vol. 8, pp. 2169-3536, 2020. DOI: 10.1109/ACCESS.2020.3007216.

[38]. J. Tang, et al., "Effect of frozen-thaw injury on cell membrane and bio-impedance," In 2020 IEEE International Instrumentation and Measurement Technology Conference (I2MTC), pp. 1-6. IEEE, 2020.

[39]. J. Tang, et al., "Bio-impedance spectroscopy for frozen-thaw of biosamples: Non-contact inductive measurement and finite element (FE) based cell modelling," Journal of Food Engineering, vol. 272, pp. 109784, 2020. 
[40]. H. Xu et al., "Imaging a weld cross-section using a novel frequency feature in multi-frequency eddy current testing," Insight-NonDestructive Testing and Condition Monitoring, vol. 61, no. 12, pp. $738-743,2019$.

[41]. Y. Xie et al., "Novel Wearable Sensors for Biomechanical Movement Monitoring Based on Electromagnetic Sensing Techniques," IEEE Sensors Journal, vol. 20, no. 2, 2020. DOI: 10.1109/JSEN.2019.2943487

[42]. H. Xu et al., "Permeability invariance phenomenon and measurement of electrical conductivity for ferrite metallic plates," Insight-Non-Destructive Testing and Condition Monitoring, vol. 61, no. 8, pp. 472 - 479, 2019.

[43]. M. Lu et al., "A model for the triboelectric nanogenerator with inductive load and its energy boost potential," Nano Energy, vol. 63, pp. 103883, 2019.

[44]. M. Lu et al., "Forward solver for deep earth exploration and induction logging using custom built Edge - Element FEM technique," Acta Geologica Sinica, vol. 93, pp. 302-304, 2019.

[45]. L. Chen et al., "Whole System Design of Wearable Magnetic Induction Sensor for Physical Rehabilitation," Advanced Intelligent Systems, vol. 1, no. 1, pp. 1900037, 2019.

[46]. Y. X et al., "A self-powered radio frequency (RF) transmission system based on the combination of triboelectric nanogenerator (TENG) and piezoelectric element for disaster rescue/relief," Nano Energy, vol. 54, pp. 331-340, 2018.

[47]. W. Yin et al., "Custom edge - element FEM solver and its application to eddy-current simulation of realistic $2 \mathrm{M}$ - element human brain phantom," Bioelectromagnetics, vol. 39, no. 8, pp. 604-616, 2018.

[48]. L. Yin et al., "Detection of corrosion pits based on an analytically optimised eddy current sensor," Insight-Non-Destructive Testing and Condition Monitoring, vol. 60, no. 10, pp. 561-567, 2018.

[49]. W. Yin et al., "Acceleration of eddy current computation for scanning probes," Insight-Non-Destructive Testing and Condition Monitoring, vol. 60, no. 10, pp. 547-555, 2018.

[50]. W. Zhou et al., "Three-dimensional electromagnetic mixing models for dual-phase steel microstructures," Applied Sciences, vol. 8, no. 4, pp. 547-555, 2018.

[51]. M. Lu, et al., "Determining the magnetic permeability of ferrite steel strip by a custom inversion method," In Proc. 12th ECNDT, pp. 1-8. 2018.

[52]. J. Tang, et al., "Cellular structure analysis based on magnetic induction finite element method simulations and measurements," bioRxiv, pp. 275271, 2018. DOI: 10.1101/275271

[53]. J.R.S. Avila, et al., "A novel dual modality sensor with sensitivities to permittivity, conductivity, and permeability," IEEE Sensors Journal, vol. 18, no. 1, pp. 356-362, 2017.

[54]. T. Yang, et al., "Level measurement for saline with a small surface area using high frequency electromagnetic sensing technique," Measurement, vol. 101, pp. 118-125, 2017.

[55]. M. Lu, et al., "Prediction of the asymptotical magnetic polarization tensors for cylindrical samples using the boundary element method," In 2015 IEEE Sensors Applications Symposium (SAS), pp. 1-4. IEEE, 2015. 